\title{
FLI1 promotes protein translation via the transcriptional regulation of MKNK1 expression
}

\author{
CHUNLIN WANG ${ }^{1,2}$, JIALEI SONG ${ }^{1,2}$, WULING LIU ${ }^{1,2}$, YAO YAO $^{1,2}$, \\ PHILIPP KAPRANOV ${ }^{3}$, KLARKE M. SAMPLE ${ }^{4}$, BABU GAJENDRAN ${ }^{1,2}$, \\ ELDAD ZACKSENHAUS ${ }^{5,6}$, XIAOJIANG HAO ${ }^{1,2}$ and YAACOV BEN-DAVID ${ }^{1,2^{*}}$
}

\author{
${ }^{1}$ State Key Laboratory for Functions and Applications of Medicinal Plants, Guizhou Medical University; \\ ${ }^{2}$ The Key Laboratory of Chemistry for Natural Products of Guizhou Province and Chinese Academic of Sciences, \\ Province Science City, High Tech Zone, Baiyun, Guiyang, Guizhou 550014; ${ }^{3}$ Institute of Genomics, School of Biomedical \\ Sciences, Huaqiao University, Xiamen, Fujian $361021 ;{ }^{4}$ Central Laboratory, Guizhou Provincial People's \\ Hospital, The Affiliated Hospital of Guizhou University Medical College, Guiyang, Guizhou 550002, P.R. China; \\ ${ }^{5}$ Department of Medicine, University of Toronto, Toronto, ON M5S 1A8; ${ }^{6}$ Division of Advanced Diagnostics, \\ Toronto General Research Institute, University Health Network, Toronto, ON M5G 2C4, Canada
}

Received July 25, 2019; Accepted November 18, 2019

DOI: 10.3892/ijo.2019.4943

\begin{abstract}
The disruption of protein translation machinery is a common feature of cancer initiation and progression, and drugs that target protein translation offer new avenues for therapy. The translation initiation factor, eukaryotic initiation factor 4E (eIF4E), is induced in a number of cancer cell lines and is one such candidate for therapeutic intervention. Friend leukemia integration 1 (FLI1) is a potent oncogenic transcription factor that promotes various types of cancer by promoting several hallmarks of cancer progression. FLI1 has recently been implicated in protein translation through yet unknown mechanisms. This study identified a positive association between FLI1 expression and mitogen-activated protein kinase (MAPK)-interacting serine/threonine kinase1 (MKNK1), the immediate upstream regulator of the eIF4E initiation factor. The short hairpin RNA (shRNA)-mediated silencing or overexpression of FLII in leukemic cell lines
\end{abstract}

Correspondence to: Professor Yaacov Ben-David, The Key Laboratory of Chemistry for Natural Products of Guizhou Province and Chinese Academic of Sciences, Province Science City, High Tech Zone, Baiyun, Guiyang, Guizhou 550014, P.R. China E-mail: yaacovbendavid@hotmail.com

Abbreviations: MAPK, mitogen-activated protein kinase; FLI1, friend leukemia integration 1, ERK, extracellular signal-regulated kinase; eIF4E, eukaryotic initiation factor 4E; FMR1, fragile $X$ mental retardation protein, MKNK, mitogen-activated protein kinase (MAPK)-interacting serine/threonine kinase; ChIP, chromatin immunoprecipitation

Key words: Friend leukemia integration 1, transcriptional regulation, mitogen-activated protein kinase-interacting serine/threonine kinase1, translation initiation, survivin, miR-145, proliferation downregulated or upregulated $M K N K 1$ expression, respectively. Promoter analysis identified a potent FLI1 binding site in the regulatory region of the $M K N K 1$ promoter. In transient transfection experiments, $F L I 1$ increased $M K N K 1$ promoter activity, which was blocked by mutating the FLI1 binding site. FLI1 specifically affected the expression of $M K N K 1$, but not that of $M K N K 2$. The siRNA-mediated downregulation of $M K N K 1$ downregulated the expression of survivin (BIRC5) and significantly suppressed cell proliferation in culture. FLI1 inhibitory compounds were shown to downregulate this oncogene through the suppression of MAPK/extracellular-regulated kinase (ERK) signaling and the subsequent activation of miR-145, leading to a lower MKNK1 expression and the suppression of leukemic growth. These results uncover a critical role for FLI1 in the control of protein translation and the importance of targeting its function and downstream mediators, such as MKNK1, for cancer therapy.

\section{Introduction}

The dysregulation of protein synthesis by oncogenes and tumor suppressor genes is a common event in cancer (1) The therapeutic targeting of the translational machinery has thus become an area of substantive interest for cancer therapy. In this respect, a number of small molecules/compounds have been discovered to target various components of the translational apparatus (2).

Mitogen-activated protein kinase (MAPK)-interacting serine/threonine kinases (MKNKs) 1/2 (MKNK1/MKNK2) control translational initiation through the phosphorylation of eukaryotic initiation factor 4E (eIF4E) $(3,4)$. MKNK1 is recruited to eIF4E through the binding of eIF4G (5). When phosphorylated, eIF4E binds to other components within the initiation complex to begin translation (6). Of note, fragile $\mathrm{X}$ mental retardation protein (FMR1) has been shown to regulate the translation of specific mRNAs through its binding 
to eIF4E (7). Higher levels of phosphorylated eIF4E have also been reported to increase the rate of development and progression of various types of cancer (6). Upstream events that activate ERK/MAPK and p38 kinases have been shown to phosphorylate residues Thr209 and Thr214 in MKNK1 and Thr244 and Thr249 in MKNK2 $(4,8,9)$. While the phosphorylation and activation of eIF4E are necessary for oncogenic transformation, knockout experiments have revealed that both MKNK1 and MKNK2 are dispensable for normal development (10). Targeting eIF4E may therefore be an effective strategy for cancer therapy with minimal side-effects (11).

Friend virus-induced Leukemia-1 (FLI1) transcription factor (TF) is a potent oncogene that drives the initiation and progression of leukemia and other types of cancer (12-15). During oncogenesis, FLI1 alters a number of hallmarks of cancer, including proliferation, apoptosis, differentiation, angiogenesis, genomic stability and inflammation (15-17). Thus, the therapeutic targeting of FLI1 is an attractive strategy. Accordingly, several classes of compounds with a potent anti-FLI1 activity have recently been identified (18-22). Among these, two flavagline-like compounds exhibiting potent FLI1 inhibition were also identified (22). These compounds suppress c-Raf-MEK-MAPK/ERK signaling, resulting in the reduced phosphorylation of eIF4E and the inhibition of FLI1 protein synthesis.

The present study, at least to the best of our knowledge, demonstrates for the first time that FLI1 is capable of binding to the promoter of $M K N K 1$, directly activating MKNK1 transcription and subsequently, the phosphorylation of eIF4E. Thus, these results highlight the use of FLI1 inhibitors for the suppression of protein translation for the treatment of cancers overexpressing this TF.

\section{Materials and methods}

Cell lines and drug treatment. Mycoplasma negative erythroleukemia cell lines (human HEL [ATCC-TIB-180] and K562 [ATCC-CCL-243]; mouse KH16, CB7 and DP17 (generated by the authors and others) $(13,18)$ were cultured and maintained in Dulbecco's Modified Eagle Medium supplemented with 5\% fetal bovine serum (HyClone, GE Healthcare).

For drug treatment, the HEL cells were treated with $3 \mu \mathrm{M}$ of A1544, A1545 (22) and CGP57380 and $24 \mathrm{~h}$ later subjected to either western blot analysis or RT-qPCR. The drug CGP57380 was obtained from Selleckchem. The drugs were dissolved to a stock solution of $2 \mathrm{mM}$ in dimethyl sulfoxide (DMSO), diluted to concentrations indicated in the text and used in treatments. DMSO was also used as a vehicle control.

The K562-fli-1 inducible cell line was generated by the authors, as previously described (18). To induce FLI1, $10^{6}$ cells were treated for $24 \mathrm{~h}$ with $10 \mathrm{nM}$ of doxycycline (Cat. no. D8960-5g, Solarbio) and used in the experiments described below.

Gene cloning, transfections and luciferase activity. To clone the $M K N K 1$ promoter, various regions of the $M K N K 1$ promoter (for details please see Fig. 2B) were isolated by qPCR (the list of primers is presented in Table SI) and cloned into the luciferase reporter vector PGL3 (Promega), as previously described (21). These promoter vectors $(1 \mu \mathrm{g})$ with either
MigR1 $(1 \mu \mathrm{g})$ or MigR1-FLI1 $(1 \mu \mathrm{g})$ were transfected into 293T cells (ATCC-CRL-3216) using Lipofectamine 2000 (Life Technologies; Thermo Fisher Scientific) following the manufacturer's protocol. Renilla luciferase was used in transfection as an internal control to examine the transfection efficiency, according to the manufacturer's recommendations (Promega). The transfected cells were then plated $8 \times 10^{3}$ cells/well into 96-well plates and luciferase activity was determined, as previously described (21).

The DP17 $\left(1 \times 10^{6}\right)$ cells were transfected with MigR1-FLI1 $(2 \mu \mathrm{g})$ and MigR1 $(2 \mu \mathrm{G})$ vector, using Lipofectamine 2000, and cells were then selected for neomycin resistance by growing the cells in medium containing G418 $(0.8 \mathrm{mg} / \mathrm{ml}$; Gibco; Thermo-Fisher Scientific).

Chromatin immunoprecipitation (ChIP) analysis. For ChIP assay, CB7 cells were washed, crosslinked with formaldehyde, resuspended in lysis buffer Magna Chip G kits (Millipore) and fixed cells sonicated using the Sonics Vibra VCX150 (Scientz Biotechnology). At this stage, a portion of chromatin aliquot was removed for input control. To the isolated chromatin protein $\mathrm{G}$ sepharose beads was added followed by incubation for $1 \mathrm{~h}$ at room temperature. Immunoprecipitations were performed overnight at $4^{\circ} \mathrm{C}$ with $1 \mu \mathrm{g}$ of FLI1 antibody (Cat. no. ab15289, Abcam) or non-specific normal rabbit immunoglobulin $\mathrm{G}$ (IgG; Cat. no. 2729, Cell Signaling Technology) antibodies. Precipitates were then washed and reverse crosslinked, using the instructions provided with the Magna Chip G kits (Millipore). Precipitated chromatin was then incubated with proteinase $\mathrm{K}$ at $50^{\circ} \mathrm{C}$ for $2 \mathrm{~h}$, DNA-purified with phenol chloroform extraction and resuspended in TE buffer. RT-qPCR was performed to amplify the $M K N K 1$ promoter regions containing FLI1 binding site 1 (position -482 to -205) and for negative ChI control (position -730 to -453 ). The sequences of the ChIP primers are presented in Table SII. The percentage of input was calculated by RT-qPCR based upon the intensity of the amplified FLII DNA divided by the amplified input DNA. Amplified DNA was also resolved on a $2 \%$ agarose gel and illustrated in Fig. 3E (right panel).

$R N A$ preparations and $R T-q P C R$. Total RNA was extracted from the growing culture of HEL cells using TRIzol reagent (Life Technologies; Thermo Fisher Scientific) according to the manufacturer's protocol. A NanoDrop 2000 spectrophotometer (Thermo Fisher Scientific) was used to determine the RNA concentration. To generate cDNA, the reverse transcription reaction was performed using the PrimeScript RT Reagent kit (Takara). qPCR was performed using FastStart Universal SYBR-Green Master (Roche) and the Step One Plus Real-time PCR system (Applied Biosystems). The expression was normalized to the $\beta$-actin level. The primer sequences are presented in Table SII. Three biological triplicates were used for all RT-qPCRs, each in triplicate $(n=3)$. The primer efficiency was calculated and is summarized in Table SII.

shRNA and siRNA transfection. The sh-FLI1 expression construct (FLI1-shRNA) was as previously described (18). The Mknk1 siRNAs (Mknk1-si1-si3) and control scrambled plasmids were purchased from (GenePharma). The sequences are presented in Table SII. The transfection of these siRNAs 

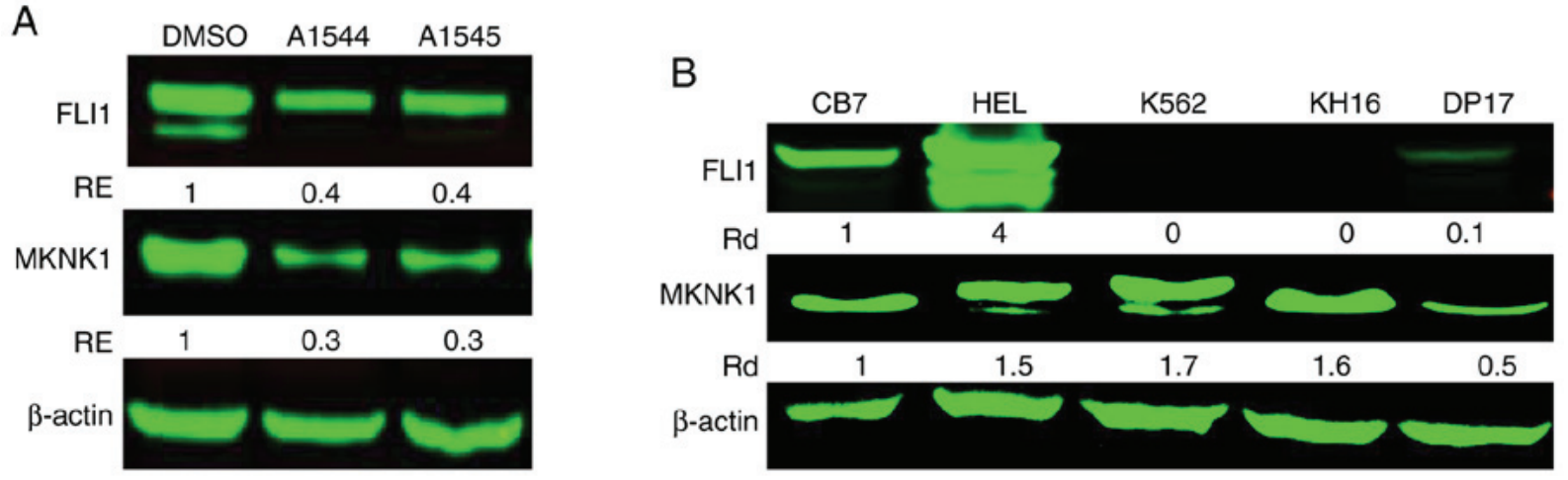

Figure 1. Association between FLI1 and MKNK1 expression in leukemic cell lines. (A) Western blot analysis showing that the downregulation of FLI1 by anti-FLI1 compounds A1544 and A1544 resulted in the suppression of MKNK1 expression in the HEL leukemia cell line. (B) Identification of a positive association between FLI1 and MKNK1 expression in the indicated cell lines. $\beta$-actin was used as a loading control. Rd, relative density; FLII, friend leukemia integration 1; MKNK1, mitogen-activated protein kinase (MAPK)-interacting serine/threonine kinase 1.

A
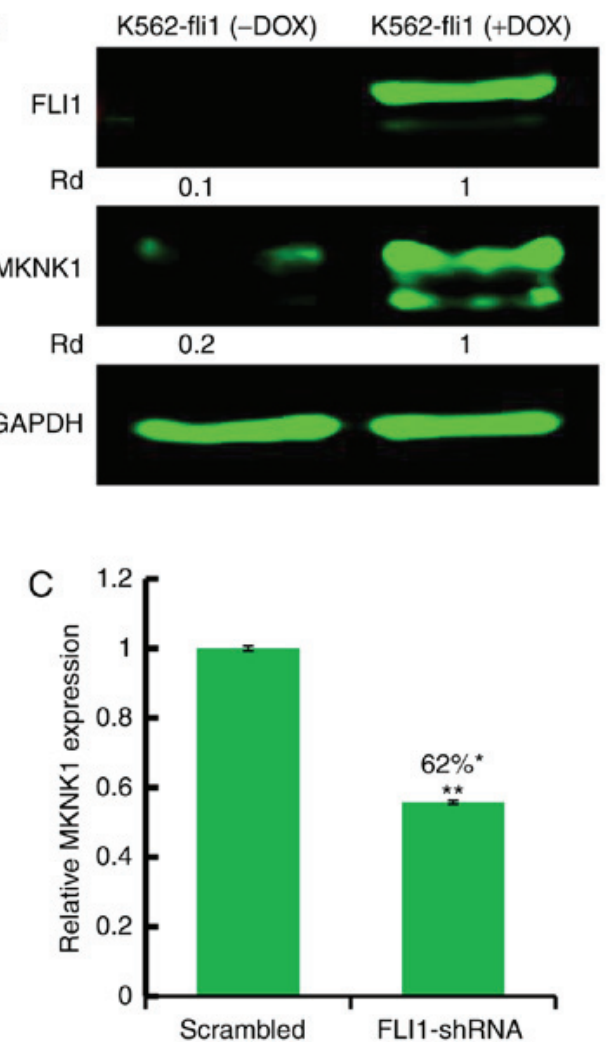

B

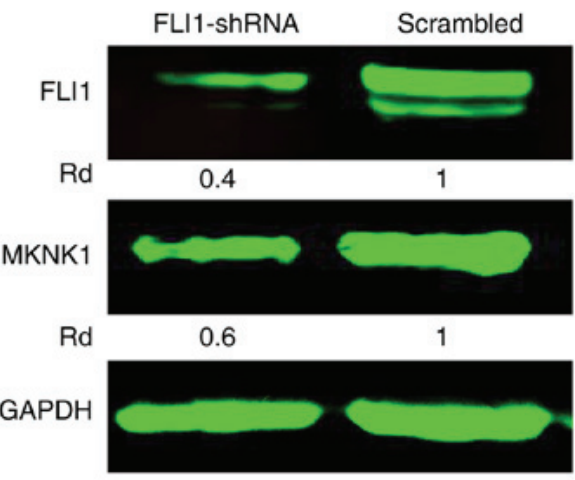

D
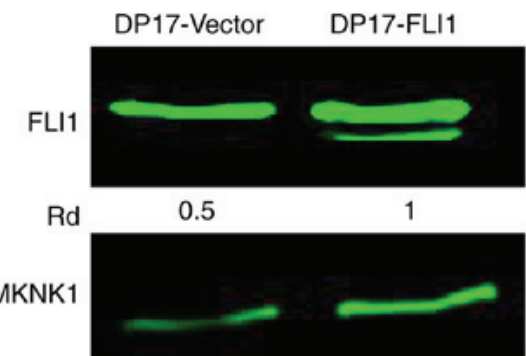

Rd

1

$\beta$-actin

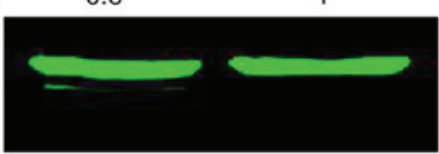

Figure 2. FLI1 modulates MKNK1 expression in leukemia cell lines. (A) In K562 cells expressing FLI1 inducible plasmid (K562-fli1), the induction of FLI1 by addition of doxycycline $(5 \mu \mathrm{M})$ for 1 day resulted in an increased MKNK1 expression. (B and C) Downregulation of FLII by FLI1-shRNA resulted in the suppression of MKNK1 (B) protein and (C) mRNA expression in HEL cells. Asterisk (*) indicates the percentage of Fli1- mRNA suppression in FLI-shRNA transfected cells. (D) Overexpression of FLI1 in DP-17 cells increased MKNK1 expression. ${ }^{* *} \mathrm{P}<0.005$. FLI1, friend leukemia integration 1; MKNK1, mitogen-activated protein kinase (MAPK)-interacting serine/threonine kinase 1.

into the HEL cells was performed using Lipofectamine 2000 according to the manufacturer's instructions (Invitrogen; Thermo Fisher Scientific), and as previously described (18).

Western blot analysis and inhibitor drugs. The procedure used for western blot analysis was as previously described $(18,23)$. Polyclonal rabbit antibodies against MKNK2 (Cat. no. ab84345), eIF4E (Cat. no. ab33766), phospho-eIF4E (Cat. no. ab76256), cMYC (Cat. no. ab39688) and FLI1 (Cat. no. ab133485) were all purchased from Abcam; MKNK1 (Cat. no. 2195) and survivin (Cat. no. 2808) antibodies were obtained from Cell Signaling Technology); GAPDH (Cat. no. G9545) antibody was obtained from Sigma-Aldrich; $\beta$-actin antibody (Cat. no. 20536-1-AP) was obtained from Proto-Technology (Protein-Tech); goat-anti-mouse and goat anti-rabbit HRP-conjugated antibodies were obtained from Cell Signaling Technology (Cat. nos. 5470s and 5151s, respectively). Primary antibodies were added to the filters 

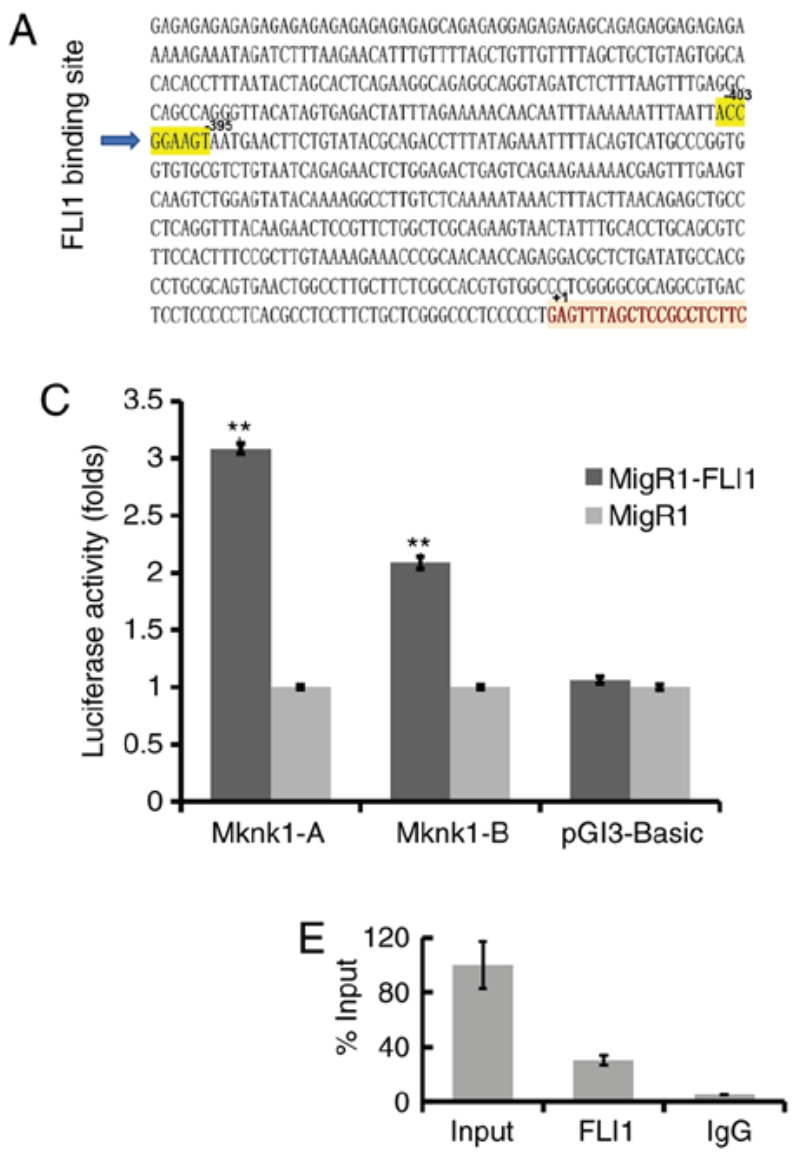
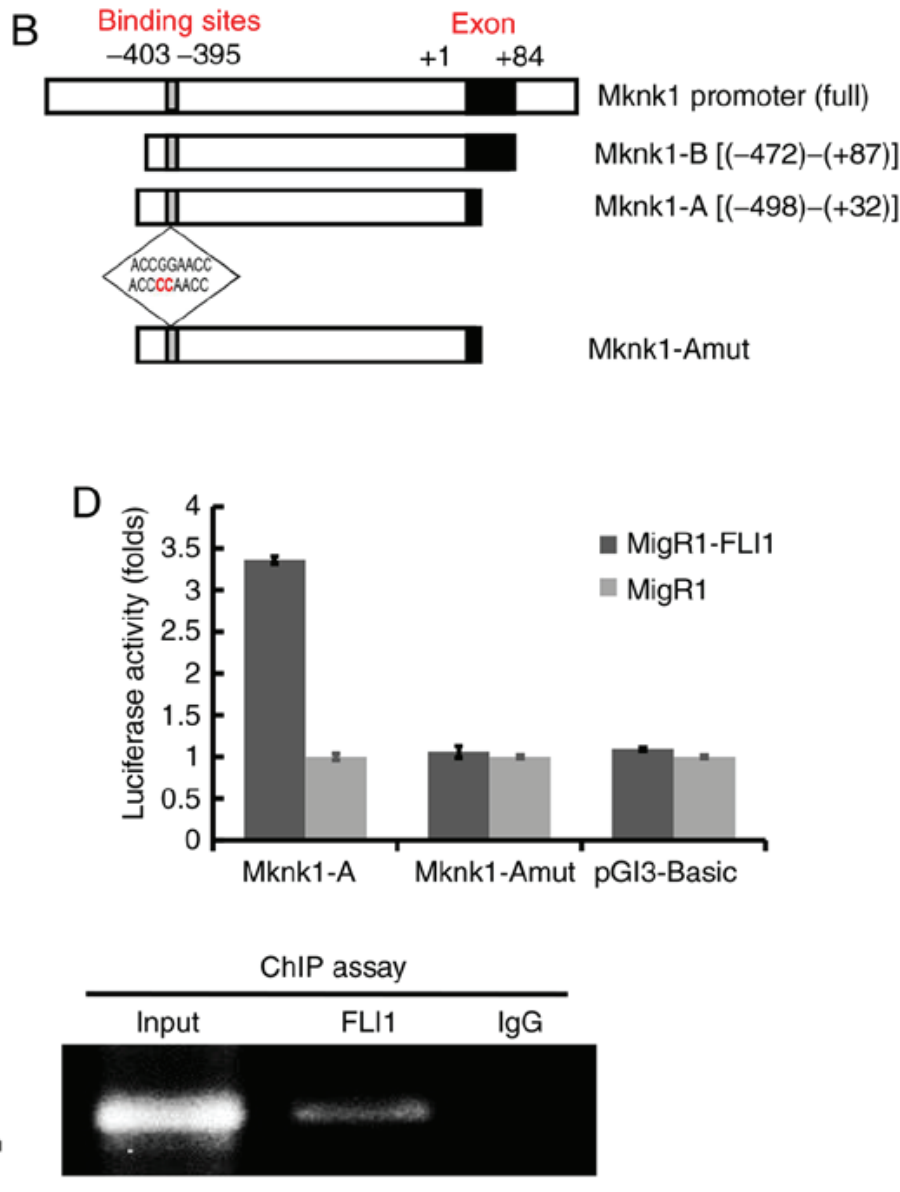

Figure 3. FLI1 positively regulates the Mknk1 promoter. (A) Murine Mknk1 gene contains a putative FLI1 binding site at nucleotide positions -403 to -395 (shown by arrow). (B) Construction of different region of the Mknkl gene upstream of the reporter plasmid PGL3. Insert shows the mutations within the FLI1 binding site in the Mknk1-A promoter. (C) Luciferase assays of indicated plasmids after transient transfection into 293T cells. (D) Luciferase activity of Mknk1-A and Mknk1-Amut when compared to PGL3-Basic vector. (E) Chromatin immunoprecipitation (ChIP) assay for the binding of murine FLI1 to a region in the Mknk1 promoter that contains the FLI1 binding site. ${ }^{* *} \mathrm{P}<0.005$. FLI1, friend leukemia integration 1; MKNK1, mitogen-activated protein kinase (MAPK)-interacting serine/threonine kinase 1.

and incubated overnight at $4^{\circ} \mathrm{C}$. After washing, secondary antibodies were added for $1 \mathrm{~h}$ at room temperature. Antibody dilution was according to the manufacturer's instructions. The Odyssey system (LI-COR Biosciences) was used to image proteins in western blot analysis.

The inhibitor of MKNK1 (CGP57380) was obtained from Selleckchem. The Fli-1 inhibitors, A1544 and A1545, were used as previously described (22).

Statistical analysis. Statistical analysis was carried out using the two-tailed Student t-test with significance considered at $\mathrm{P}<0.05$, and by one-way ANOVA with Tukey's post-hoc test, using Origin 3.5 software (Microcal Software). The results were expressed as the means \pm standard deviation from at least 3 independent experiments.

\section{Results}

FLI1 expression is associated with MKNK1 expression in leukemic cell lines. The authors have previously reported that the anti-FLI1 A1544 and A1545 compounds downregulate the phosphorylation of eIF4E in leukemic cell lines (22). Accordingly, in this study, the expression of MKNK1, an upstream regulator of eIF4E, was found to be lower in the HEL cells treated with the A1544 and A1545 compounds (3 $\mu \mathrm{M})$, compared to the control DMSO-treated cells (Fig. 1A). This and related analyses raised the possibility that FLI1 may regulate $M K N K 1$ expression. Indeed, in the cell lines expressing FLI1 (HEL, CB7 and DP17), the expression of this oncogene was positively associated with MKNK1 expression (Fig. 1B). In the FLI1-negative cell lines, K562 and KH16, MKNK1 expression was relatively high, indicating different mechanisms of gene regulation. However, when FLI1 expression was induced by doxycycline in the K562 cells transfected with a doxycycline inducible promoter (18), the increased FLI1 expression resulted in a higher MKNK1 protein (Fig. 2A) and mRNA (Fig. S1) expression compared to the non-induced control cells (Fig. 2A). In HEL cells expressing high levels of FLI1, the anti-FLII shRNA-mediated silencing resulted in a lower MKNK1 protein abundance (Fig. 2B) and mRNA (Fig. 2C) expression. Moreover, in murine DP17 cells with a lower FLI1 expression, the exogenous expression of FLI1 markedly increased MKNK1 expression (Fig. 2D). Overall, these data strongly suggest that FLI1 regulates MKNK1 expression in both murine and human cells. 

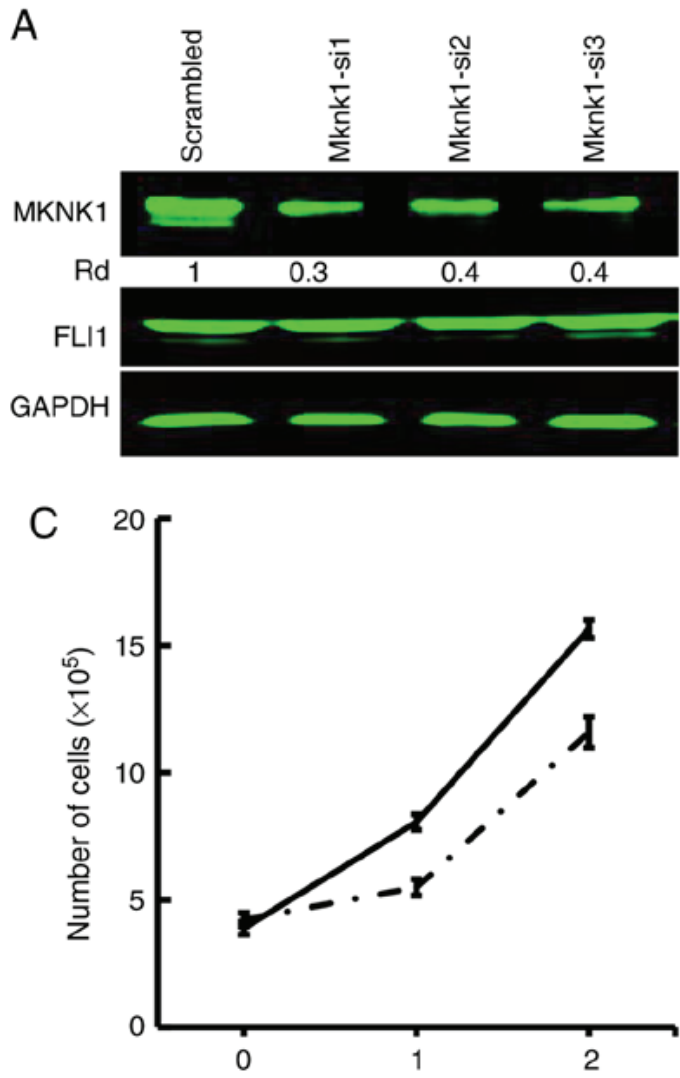

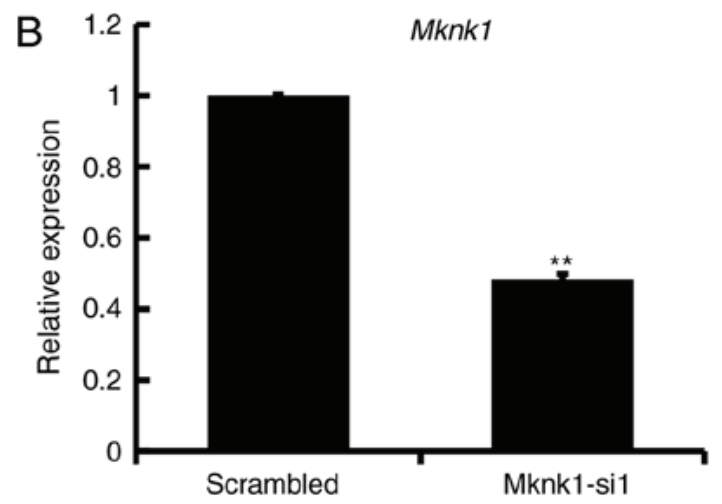

$\mathrm{D}$

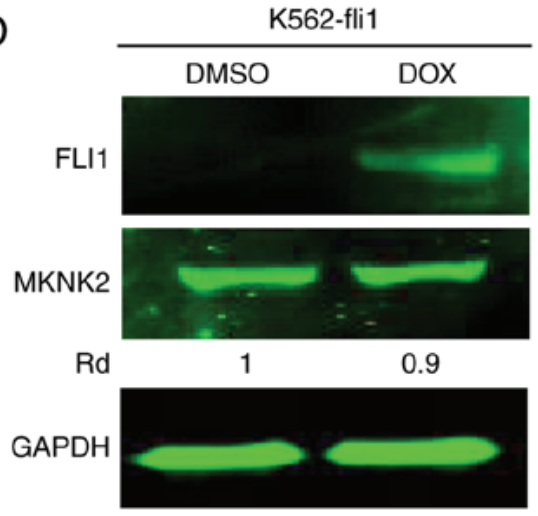

Figure 4. $M K N K 1$ downregulation inhibits the proliferation of leukemic cells. (A) $M K N K 1$ downregulation using 3 siRNA constructs (Mknk1-si1-Mknk1-si3), detected by western blot analysis. (B and C) In HEL cells, the (B) downregulation of $M K N K 1$ by Mknk1-si1, (C) resulted in significant growth inhibition in culture. (D) FLI1 overexpression in K562 (K562-fli1) cells by doxycycline increased FLI1 level, but exhibited a marginal effect on the expression of MKNK2. GAPDH was used as a loading control. ${ }^{* *} \mathrm{P}<0.005$. FLI1, friend leukemia integration 1; MKNK, mitogen-activated protein kinase (MAPK)-interacting serine/threonine kinase.

FLII binds to the murine Mknkl promoter and positively regulates its expression. The promoter of murine $M k n k l$ contains a putative FLI1 binding site (ACCGGAAGT) at position -403 to -395 (Fig. 3A). Fragments of the $M k n k 1$ promoter regions (designated as Mknk-A and Mknk-B) were subcloned upstream of the luciferase reporter plasmid PGL3 (Fig. 3B). These promoters were transfected with FLI1 (MigR1-FLI1) or control (MigR1) expression vectors into 293T cells, examined for FLI1 expression (Fig. S2) and later for luciferase activity. The Mknk-A promoter fragment displayed a significantly higher luciferase activity than the other constructs (Fig. 3C). The putative FLI1 binding site within Mknk-A was subsequently mutated (Fig. 3B, bottom insert) and tested for luciferase activity. The mutation of the FLI1 binding site (ACCGGAAGT to ACCCCAAGT) within Mknk-A completely blocked promoter activity (Fig. 3D). ChIP assay using FLI1 antibody and control $\mathrm{IgG}$ was performed on chromatin fragments of the $m k n k l$ promoter isolated from sheared genomic DNA of mouse CB7 cells. This analysis revealed significant binding of FLI1 to the Mknkl promoter having a Fli-1 binding site (FBS), relative to input (Fig. 3E). This binding was not observed by ChIP analysis in the immediate upstream region of the $M k n k l$ promoter (designated FBS-NC), lacking FLI1 binding site (Fig. S3 and Table SII). These results demonstrate that the murine $M k n k l$ promoter is a direct target of FLI1.
siRNA-mediated MKNK1 silencing suppress the proliferation of leukemic cells in culture. To examine the effects of MKNK1 expression on proliferation, siRNA was used to silence the gene in HEL cells. All 3 siRNAs (Mknk1-si1, Mknk1-si2 and Mknk1-si3) markedly suppressed MKNK1 expression (Fig. 4A); Mknk1-si1 was used in the subsequent experiments. The siRNA-mediated downregulation of $M K N K 1$, as detected by western blot analysis and RT-qPCR (Fig. 4A and B), resulted in a significant suppression of proliferation compared to the scrambled control-transfected cells (Fig. 4C). Of note, the induction of FLI1 in K562-fli1 inducible cells did not increase the level of MKNK2 (Fig. 4D). Since MKNK1 was induced by FLI1 in this system (Fig. 2A) and downregulated by shFLI1 (Fig. 2B), this result excluded MKNK2 as a target of FLI1 (Fig. 4D). Thus, while MKNK1 downregulation clearly inhibited cell proliferation, FLI1 altered cell proliferation in part through the specific upregulation of this gene.

Regulation of survivin (BIRC5) by FLI1 through the MKNK1 pathway. Previous studies have suggested that MYC (cMYC) expression is downstream of the MKNK1 pathway $(22,24)$. However, in this study, while the anti-FLI1 compounds, A1544 and A1545, and the specific MKNK1 inhibitor, CGP57380, markedly inhibited MKNK1 and downstream phospho-eIF4A expression, the level of MYC remained unaltered by treatment with A1544 and A1545 (Fig. 5A). Indeed, a significant increase in MYC expression was observed in the CGP57380-treated 


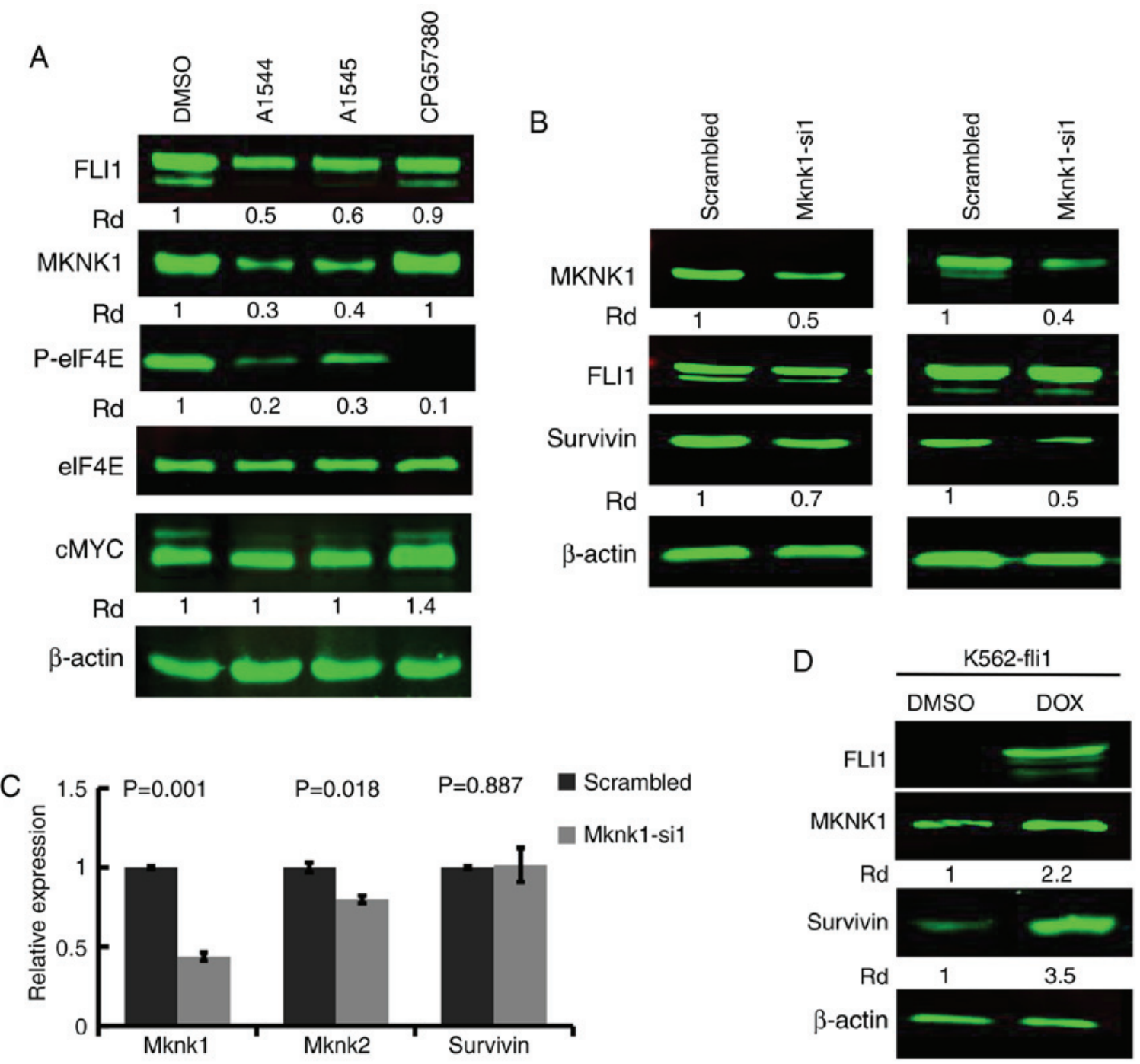

Figure 5. MKNK1 downregulation inhibited survivin expression in leukemic HEL cells. (A) Treatment of HEL cells with A1542, A1543 or CGP57380 compounds ( $3 \mu \mathrm{M})$ downregulated MKNK1 and phospho-eIF4E, but not cMYC expression. (B) MKNK1 silencing by Mknk1-sil in 2 independent experiments significantly decreased (B) the expression of survivin protein, (C) but not its transcription, as detected by RT-qPCR, compared to scrambled control transfected cells. (D) FLI1 overexpression in K562 (K562-fli1) cells by doxycycline increased both MKNK1 and survivin expression. FLI1, friend leukemia integration 1; MKNK, mitogen-activated protein kinase (MAPK)-interacting serine/threonine kinase.

HEL cells. This observation excluded MYC translational regulation by MKNK1 in these cells.

The FLI1 inhibitors, A1544 and A1545, have previously shown to inhibit anti-apoptotic protein BIRC5 expression through the suppression of eIF4E (22), in which the high expression of this protein in leukemia cells facilitates cell growth $(22,25)$. In this study, since eiF4E phosphorylation and its activity were regulated by MKNK1, the si1-mediated silencing of $M K N K 1$ in 2 independent experiments resulted in a marked decrease in the expression of BIRC5 protein (Fig. 5B), but not its mRNA expression (Fig. 5C). Notably, in this study, MKNK2 expression was slightly downregulated, although the level of $M K N K 1$ was significantly decreased (Fig. 5C). As predicted, induction of FLI1 in K562 inducible fli1 cells by doxycycline significantly increased survivin expression (Fig. 5D). Moreover, the upregulation of survivin expression following induction by doxycycline in K562-fli1 cells was partially blocked through the si1-mediated downregulation of Mknk1 (Fig. S4). These results demonstrate the regulation of BIRC5 by the FLI1/MKNK/eIF4E pathway.
Association between miR-145, FLII and MKNK1 in regulating protein translation in leukemic cells. Previous studies by the authors, as well as others have revealed a negative regulation of the miR-145 promoter by FLI1 $(21,26-29)$, and the inhibition of FLI1 mRNA translation by miR-145 (26), establishing a negative regulatory loop $(21,26)$. The anti-FLI1 compounds A1544 and A1545 have been previously shown to target the proto-oncogene serine/threonine-protein kinase c-RAF for inhibition, leading to suppression of MAPK/ERK and inhibition of FLI1 translation (22). However, the mechanisms through which MAPK/ERK activation controls FLI1 translation remain unknown. In this study, it was demonstrated that both the A1544 and A1545 compounds significantly increased the level of miR-145 in the HEL cells (Fig. 6A). Since MAPK/ERK activation blocks miR-145 expression (30), A1544 and A1545 likely suppress FLI1 translation and subsequently MKNK1 expression through miR145 upregulation (Fig. 6B). These results emphasize the important role played by FLI1 in the regulation of translation through both miR-145 and MKNK1. 


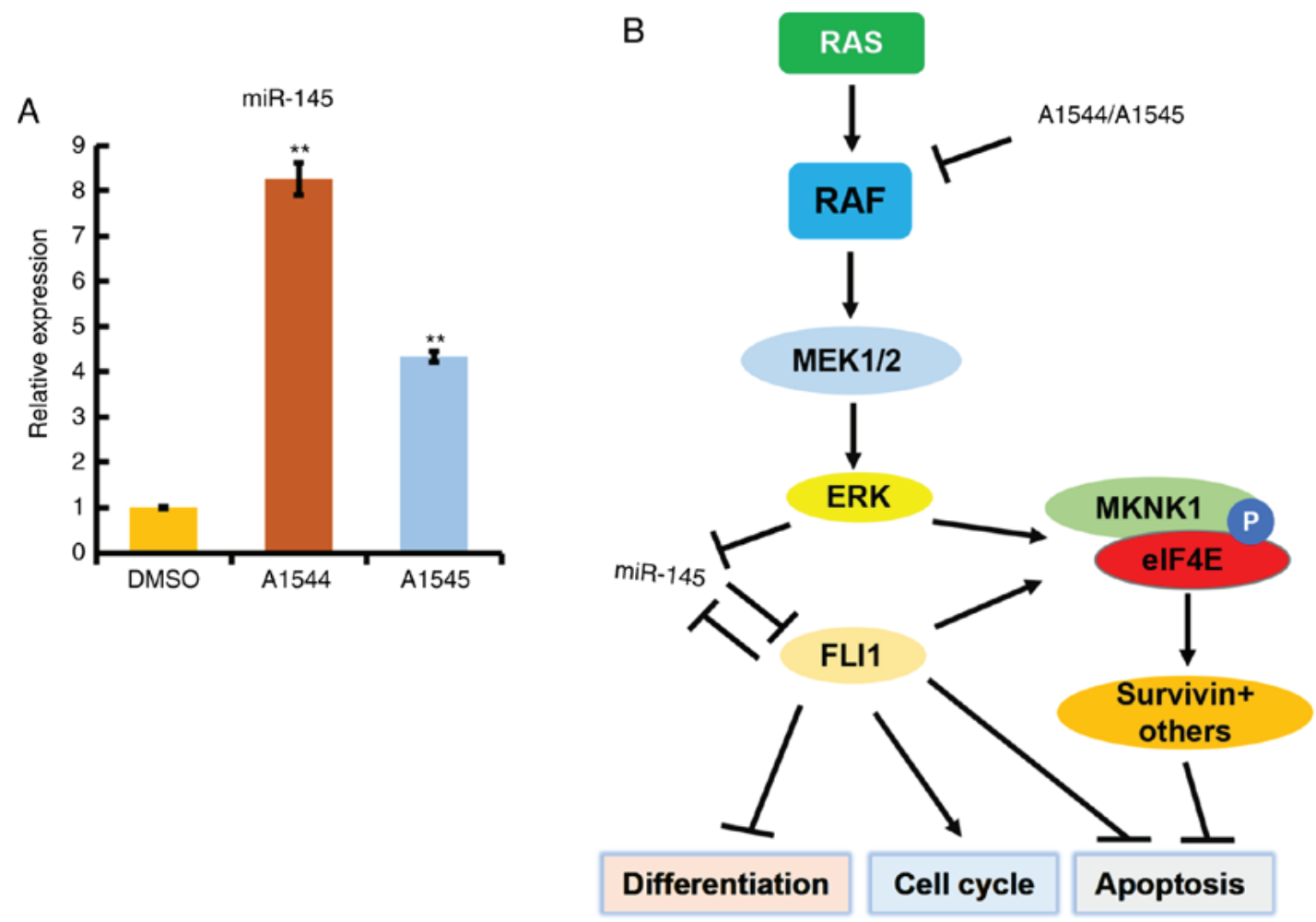

Figure 6. Anti-FLI1 compounds inhibit FLI1 expression through the upregulation of miR-145. (A) Treatment of HEL cells with the indicated drugs (3 $\mu \mathrm{M})$ resulted in upregulation of miR145, as detected by RT-qPCR. "* P<0.005. (B) A model of FLI1 regulation and its downstream effectors. The inhibition of RAF by anti-FLI1 compounds A1544 and A1545 resulted in the suppression of MAPK/ERK, as well as FLI1 protein expression. MAPK/ERK inhibition by the compounds upregulate miR-145 whose expression negatively regulates FLI1 protein expression. FLI1 downregulation, as well as suppression of MAPK/ERK, both then reduce expression of MKNK1, the phosphorylation of eIF4E and downstream survivin. FLII downregulation by A1544 and A1545 compounds alters the expression of various target genes associated with the induction of differentiation, cell cycle and apoptosis, as previously described (15). FLI1, friend leukemia integration 1; MKNK, mitogen-activated protein kinase (MAPK)-interacting serine/threonine kinase; MAPK, mitogen-activated protein kinase; ERK, extracellular signal-regulated kinase.

\section{Discussion}

FLI1 has emerged as a potent oncogene in the development of various types of cancer through the regulation of multiple downstream pathways. Although protein translation has been implicated in FLI1 tumorigenesis, the underlying mechanisms have not yet been elucidated. To the best of our knowledge, this study identified for the first time the translation initiator regulator gene $M K N K 1$ as a target of FLI1. This study demonstrates that FLI1 binds a specific site in the Mknkl promoter to activate its transcription. This in turn confers proliferative advantages to leukemic cells. Survivin (BIRC5) was identified as a downstream target of the MKNK1/eIF4E pathway, the expression of which is known to be involved in cell survival and proliferation (31). These data further emphasize the importance of targeting FLII or its downstream genes for cancer therapy.

The overexpression of FLI1 in both human and murine cells clearly resulted in a higher MKNK1 expression. While a canonical FLI1 binding site was identified in the vicinity of the transcription start site in the mouse Mknkl promoter, the location of this binding site in humans remains to be determined in future studies. However, in human leukemic cells, the downregulation of FLI1 by shRNA, drugs or its overexpression, resulted in the significant reduction or increase in the expression of MKNK1, respectively. These results strongly suggest a direct regulation of the Mknkl gene by FLI1 in both mice and humans.
In gene knockout experiments, both $M k n k 1$ and $M k n k 2$ have been found to be dispensable for normal development, although higher levels of both genes in various cancer cells are associated with growth properties $(10,11)$. While $M K N K 2$ is not a direct target of FLI1, the loss or overexpression of $M K N K 1$ appears to have small or marginal effect on the expression of $M K N K 2$, respectively. Moreover, the inhibition of $M K N K 1$ expression by either a FLI1 inhibitor (22) or siRNA significantly suppressed leukemic cell proliferation. These results demonstrate that targeting MKNK1/eIF4E or its upstream regulators, such as the MAPK pathway and FLI1, may be a potent approach for cancer therapy, particularly for cancers which are driven by FLI1.

A previous study by the authors demonstrated the suppression of phospho-eIF4E by anti-FLI1 compounds through both the inhibition of MAPK/ERK and the downregulation of FLII (22). MYC and cyclin D1 (CCNDI) are two genes reported to be regulated by MKNK1/eIF4E in multiple myelomas $(22,24)$ However, the findings of this study clearly revealed that the expression of MYC was not altered through the suppression of MKNK1/eIF4E activity. However, BIRC5 protein expression, but not its transcription, was found to be downregulated when MKNK1 expression was suppressed either by anti-FLI1 compounds or siRNA; or upregulated when FLI1 was overexpressed in leukemic cells. Survivin is a member of the inhibitor of apoptosis (IAP) family of proteins 
that play a critical role in suppressing apoptosis and regulating the cell cycle (31). Due to these properties, survivin has been identified as an important target for cancer therapy (32). The identification of other genes that are directly regulated by the MKNK1/eIF4E pathway will further increase our understanding of tumor progression by FLI1 and MKNK1.

In conclusion, in this study, $M K N K 1$ was identified as a downstream target of FLII, which can alter the translation of specific downstream targets, leading to tumor progression. Survivin was identified as a potential target of MKNK1; however, the existence of other factors that further orchestrate protein synthesis and cancer progression by FLI1 is also possible and thus further studies are warranted to fully investigate this matter.

\section{Acknowledgements}

Not applicable.

\section{Funding}

This study was supported by the Science and Technology Department of Guizhou Province innovation and project grants (6012-4001), research grants from the Thousand Talent Program of China (WQ20135200171), the 100 Leading Talents of Guizhou Province and The Natural National Science Foundation of China (21867009 and U1812403) to YBD.

\section{Availability of data and materials}

All data and materials are available without restriction. Researchers can obtain data by contacting the corresponding authors.

\section{Authors' contributions}

CW, WL, YY, BG, XH, EZ and JS contributed to the conception, design of the study, as well as data acquisition and interpretation. PK and KMS were involved in data and statistical analysis. CW drafted the manuscript. YBD, XH, PK, EZ and JS reviewed the manuscript critically. YBD supervised and also conceived and designed the study. All authors contributed to the interpretation of the findings, and reviewed, edited and approved the final manuscript.

\section{Ethics approval and consent to participate}

Not applicable.

\section{Patient consent for publication}

Not applicable.

\section{Competing interests}

The authors declare that they have no competing interests.

\section{References}

1. Bhat M, Robichaud N, Hulea L, Sonenberg N, Pelletier J and Topisirovic I: Targeting the translation machinery in cancer. Nat Rev Drug Discov 14: 261-278, 2015.
2. Chu J and Pelletier J: Therapeutic opportunities in eukaryotic translation. Cold Spring Harb Perspect Biol 10: a032995, 2018.

3. Waskiewicz AJ, Johnson JC, Penn B, Mahalingam M, Kimball SR and Cooper JA: Phosphorylation of the cap-binding protein eukaryotic translation initiation factor $4 \mathrm{E}$ by protein kinase Mnk1 in vivo. Mol Cell Biol 19: 1871-1880, 1999.

4. Waskiewicz AJ, Flynn A, Proud CG and Cooper JA: Mitogen-activated protein kinases activate the serine/threonine kinases Mnk1 and Mnk2. EMBO J 16: 1909-1920, 1997.

5. Pyronnet S, Imataka H, Gingras AC, Fukunaga R, Hunter T and Sonenberg N: Human eukaryotic translation initiation factor $4 \mathrm{G}$ (eIF4G) recruits mnk1 to phosphorylate eIF4E. EMBO J 18: 270-279, 1999.

6. Siddiqui N and Sonenberg N: Signalling to eIF4E in cancer. Biochem Soc Trans 43: 763-772, 2015.

7. Napoli I, Mercaldo V, Boyl PP, Eleuteri B, Zalfa F, De Rubeis S, Di Marino D, Mohr E, Massimi M, Falconi M, et al: The fragile $\mathrm{X}$ syndrome protein represses activity-dependent translation through CYFIP1, a new 4E-BP. Cell 134: 1042-1054, 2008.

8. Jauch R, Jäkel S, Netter C, Schreiter K, Aicher B, Jäckle H and Wahl MC: Crystal structures of the Mnk2 kinase domain reveal an inhibitory conformation and a zinc binding site. Structure 13: 1559-1568, 2005.

9. Jauch R, Cho MK, Jäkel S, Netter C, Schreiter K, Aicher B Zweckstetter M, Jäckle H and Wahl MC: Mitogen-activated protein kinases interacting kinases are autoinhibited by a reprogrammed activation segment. EMBO J 25: 4020-4032, 2006.

10. Ueda T, Watanabe-Fukunaga R, Fukuyama H, Nagata S and Fukunaga R: Mnk2 and Mnk1 are essential for constitutive and inducible phosphorylation of eukaryotic initiation factor $4 \mathrm{E}$ but not for cell growth or development. Mol Cell Biol 24: 6539-6549, 2004.

11. Diab S, Kumarasiri M, Yu M, Teo T, Proud C, Milne R and Wang S: MAP kinase-interacting kinases-emerging targets against cancer. Chem Biol 21: 441-452, 2014.

12. Ben-David Y and Bernstein A: Friend virus-induced erythroleukemia and the multistage nature of cancer. Cell 66: 831-834, 1991.

13. Ben-David Y, Giddens EB and Bernstein A: Identification and mapping of a common proviral integration site Fli-1 in erythroleukemia cells induced by Friend murine leukemia virus. Proc Natl Acad Sci USA 87: 1332-1336, 1990.

14. Ben-David Y, Giddens EB, Letwin K and Bernstein A: Erythroleukemia induction by Friend murine leukemia virus: Insertional activation of a new member of the ets gene family, Fli-1, closely linked to c-ets-1. Genes Dev 5: 908-918, 1991.

15. Li Y, Luo H, Liu T, Zacksenhaus E and Ben-David Y: The ets transcription factor Fli-1 in development, cancer and disease. Oncogene 34: 2022-2031, 2015.

16. Lou N, Lennard Richard ML, Yu J, Kindy M and Zhang XK: The Fli-1 transcription factor is a critical regulator for controlling the expression of chemokine C-X-C motif ligand 2 (CXCL2). Mol Immunol 81: 59-66, 2017.

17. Sato $\mathrm{S}$ and Zhang XK: The Friend leukaemia virus integration 1 (Fli-1) transcription factor affects lupus nephritis development by regulating inflammatory cell infiltration into the kidney. Clin Exp Immunol 177: 102-109, 2014.

18. Liu T, Yao Y, Zhang G, Wang Y, Deng B, Song J, Li X, Han F, Xiao X, Yang J, et al: A screen for Fli-1 transcriptional modulators identifies PKC agonists that induce erythroid to megakaryocytic differentiation and suppress leukemogenesis. Oncotarget 8: 16728-16743, 2017.

19. Cui JW, Vecchiarelli-Federico LM, Li YJ, Wang GJ and Ben-David Y: Continuous Fli-1 expression plays an essential role in the proliferation and survival of F-MuLV-induced erythroleukemia and human erythroleukemia. Leukemia 23: 1311-1319, 2009.

20. Li YJ, Zhao X, Vecchiarelli-Federico LM, Li Y, Datti A, Cheng Y and Ben-David Y: Drug-mediated inhibition of Fli-1 for the treatment of leukemia. Blood Cancer J 2: e54, 2012

21. Liu T, Xia L, Yao Y, Yan C, Fan Y, Gajendran B, Yang J, Li YJ, Chen J, Filmus J, et al: Identification of diterpenoid compounds that interfere with Fli-1 DNA binding to suppress leukemogenesis. Cell Death Dis 10: 117, 2019.

22. Song J, Yuan C, Yang J, Liu T, Yao Y, Xiao X, Gajendran B, $\mathrm{Xu}$ D, Li YJ, Wang C, et al: Novel flavagline-like compounds with potent Fli-1 inhibitory activity suppress diverse types of leukemia. FEBS J 285: 4631-4645, 2018.

23. Vecchiarelli-Federico LM, Liu T, Yao Y, Gao Y, Li Y, Li YJ and Ben-David Y: Fli-1 overexpression in erythroleukemic cells promotes erythroid de-differentiation while Spi-1/PU.1 exerts the opposite effect. Int J Oncol 51: 456-466, 2017. 
24. De Benedetti A and Graff JR: eIF-4E expression and its role in malignancies and metastases. Oncogene 23: 3189-3199, 2004.

25. Cong XL and Han ZC: Survivin and leukemia. Int J Hematol 80 : 232-238, 2004.

26. Ban J, Jug G, Mestdagh P, Schwentner R, Kauer M, Aryee DN, Schaefer KL, Nakatani F, Scotlandi K, Reiter M, et al: Hsa-mir-145 is the top EWS-FLI1-repressed microRNA involved in a positive feedback loop in Ewing's sarcoma. Oncogene 30: 2173-2180, 2011

27. Wu P, Liang J, Yu F, Zhou Z, Tang J and Li K: miR-145 promotes osteosarcoma growth by reducing expression of the transcription factor friend leukemia virus integration 1 . Oncotarget 7: 42241-42251, 2016.

28. Zhang J, Guo H, Zhang H, Wang H, Qian G, Fan X, Hoffman AR, $\mathrm{Hu} \mathrm{JF}$ and Ge S: Putative tumor suppressor miR-145 inhibits colon cancer cell growth by targeting oncogene Friend leukemia virus integration 1 gene. Cancer 117: 86-95, 2011.
29. Larsson E, Fredlund Fuchs P, Heldin J, Barkefors I, Bondjers C, Genové G, Arrondel C, Gerwins P, Kurschat C, Schermer B, et al: Discovery of microvascular miRNAs using public gene expression data: miR-145 is expressed in pericytes and is a regulator of Fli1. Genome Med 1: 108, 2009.

30. Wang S, Liu JC, Ju Y, Pellecchia G, Voisin V, Wang DY, Leha LR, Ben-David Y, Bader GD and Zacksenhaus E: microRNA-143/145 loss induces Ras signaling to promote aggressive Pten-deficient basal-like breast cancer. JCI Insight 2: 93313, 2017.

31. Wheatley SP and Altieri DC: Survivin at a glance. J Cell Sci 132: jcs223826, 2019.

32. Garg H, Suri P, Gupta JC, Talwar GP and Dubey S: Survivin: A unique target for tumor therapy. Cancer Cell Int 16: 49, 2016.

(i) (9) This work is licensed under a Creative Commons Attribution-NonCommercial-NoDerivatives 4.0 International (CC BY-NC-ND 4.0) License. 